\title{
From Service Clouds to User-centric Personal Clouds
}

\author{
L. Ardissono, A. Goy, G. Petrone and M. Segnan \\ Dipartimento di Informatica \\ Università di Torino \\ Torino, Italy \\ \{liliana,goy,giovanna,marino\}@di.unito.it
}

\begin{abstract}
We present the Personal Cloud Platform (PCP) for the management of service clouds providing the user with a unified environment for handling her/his activities and collaborations. Within a personal cloud, the PCP enables the definition of global collaboration groups and a holistic management of the workspace awareness, concerning all the integrated services. Moreover, being based on an open architecture, the PCP supports the integration of external applications, selected by the enterprise user.
\end{abstract}

Keywords- service cloud; workspace awareness; contextawareness; holistic awareness information management

\section{INTRODUCTION (HEADING 1)}

With the large availability of broadband internet and wireless access, users are increasingly exploiting remote services in their everyday life, as well as at work. A consequence of this trend is the growing demand for unified environments supporting a holistic management of user activities and collaborations. For instance, there is an emerging need to integrate electronic calendars, possibly shared among groups of people, in order to provide the user with a unified point of view on her/his work, family schedules, and other types of commitments [11]. Moreover, the success of Web sites such as Facebook and Twitter shows the need for environments supporting different types of interaction, such as document sharing, synchronous and asynchronous communication.

Various collaboration environments have been developed which offer integrated Instant Messaging, e-mail, Calendar management, and document sharing applications, and are accessible from desktop and mobile terminals; e.g., [3]. Furthermore, the concept of Web Desktop is attracting attention, as a way to enable the user to organize her/his personal workspace by including the business services (s)he needs; e.g., [17]. However, such environments are based on vertical architectures and they can only be configured to include a pre-defined set of applications. Thus, they cannot be customized to meet the needs of an individual community that might have to use services devoted to the management of specific activities.

With the growing adoption of Service Oriented Architectures, the provision of Software as a Service and the management of software clouds integrating remote services are becoming a reality. However, at the current stage, the benefits for the individual user are limited because such clouds do not manage a context-dependent synchronization of applications. Specifically, the software cloud is conceived either as a platform offering a pre-defined set of partially integrated services, or as a framework which supports the invocation of applications working on separate data spaces; e.g., see the Amazon and Google platforms, and the Gladinet one [10], respectively. Thus, even though some services offer complementary functions, they are accessed as more or less isolated applications, which cannot provide the user with a unified access to her/his business data and activities.

In order to support the user in her/his everyday activities and collaborations, we introduce the concept of personal cloud as an infrastructure providing an abstraction level over the individual applications composing it. The Web Desktop managed within a personal cloud must be an access point to a unified environment offering complementary functions, instead of linking separate applications and workspaces. A key feature is thus the provision of unified access and awareness support across multiple collaborations. ${ }^{1}$ Specifically, the information about the community users, the existing collaboration groups, and the events occurring in such groups must be handled and presented from the viewpoint of the individual users.

In this paper, we describe the Personal Cloud Platform (PCP): this is an open platform for the management of service clouds offering a structured and integrated view of the user's activities and collaborations. The PCP includes a core set of software components supporting the definition of collaboration groups and a holistic management of the workspace awareness, concerning all the integrated applications. Specifically, the PCP supports the presentation of the awareness information to the users, suitably structured on the basis of the collaborations to which it belongs. Moreover, we are extending the PCP in order to customize the awareness support, depending on the user's notification preferences. As the PCP is based on an open architecture, the enterprise customer can integrate external applications, in order to provide the cloud users with specific functions.

This paper also describes a prototype personal cloud, based on the PCP, which we developed by integrating some widely used business applications supporting document sharing and calendar management.

${ }^{1}$ The concept of personal cloud should not be confused with that of private cloud, which concerns the management of service clouds within a private communication network. 
The remainder of this paper is organized as follows: Section 2 discusses the issue we address. Section 3 describes the concepts underlying the management of personal cloud. Section 4 shortly presents the synchronization framework underlying the PCP. Section 5 describes the PCP and our prototype personal cloud. Section 6 provides some technical details. Section 7 positions our work in the related research and Section 8 closes the paper.

\section{THE PROBLEM}

Cloud computing is emerging as a very powerful platform for the exploitation of heterogeneous resources and applications, thanks to the abstraction from their execution environment and physical location. However, with the exception of the process-based environments for the development of composite applications (e.g., see WS-BPEL [18]), it has not yet provided a satisfactory answer to the synchronization of applications in a shared context. For example, many private and public clouds offer complementary services which cannot share contact lists, awareness information, and other relevant context data, unless they are strictly related, as some Google Apps. Thus, a service cloud supports the management of the Web Desktop as a single access point to a set of isolated services, which the user must separately configure in the attempt to reconstruct her/his personal workspace.

For instance, as shown in Figure 1, the user can have to re-define all her/his collaboration groups (g1 and g2) for each application (s)he wants to use (app1 and app2). Moreover, (s)he has to separately set her notification preferences and filters for each application.

Unfortunately, this surface-level integration only supports the management of a fragmented workspace, where the context information common to the various applications cannot be synthesized to assist the user's activities. For instance, each business service manages the awareness information concerning the activities it supports, thus generating its own notification stream.

Figure 1 depicts this situation: as app1 and app2 separately propagate the awareness information to the users, these receive two interleaved and independent information streams. This means, e.g., that if a user exploits different services to carry out the activities of a certain project, (s)he is not provided with a single access point to the events occurred in the project. Conversely, (s)he has to separately track the messages it receives, and to visit the awareness spaces managed by the individual services, in order to retrieve the information.

Notice that this lack of integration is particularly problematic in collaboration scenarios. In fact, as largely discussed, users handle multiple collaborations, both at work and in their private life, and they often switch among them; e.g., see $[11,13,8]$. As each focus shift requires resuming the context of the users' activities, it has a possible disruptive effect on their attention and emotional state [15], which can only be mitigated by supporting the context resumption.

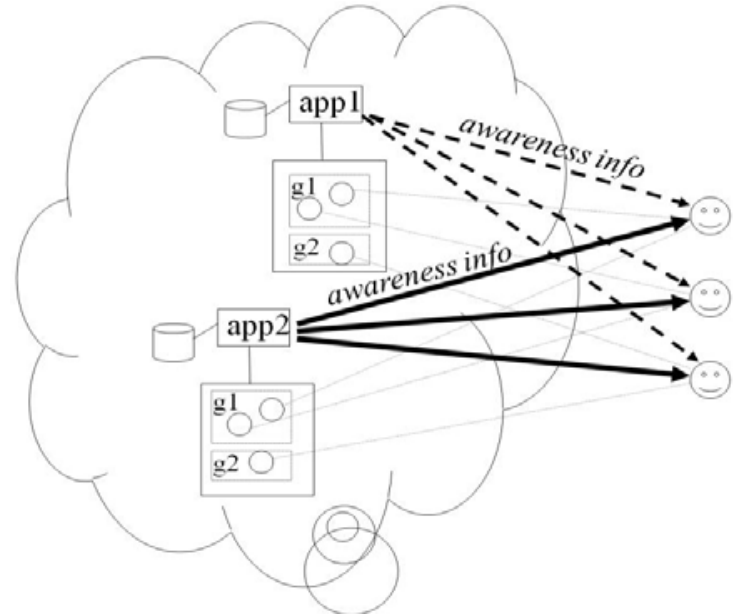

Figure 1. Separate awareness support in a service cloud.

\section{BuIlding Blocks of A PERSONAL ClOUd}

In the development of an environment supporting the management of a personal cloud, two perspectives have to be taken into account:

- The first one is the subjective viewpoint of the user, who would benefit from managing all her/his tasks in the same environment, and from receiving a holistic awareness support. For instance, the Web Desktop should enable the user to customize her/his workspace, e.g., by including the applications (s)he wants to use and her/his collaboration groups. Moreover, the Web Desktop should provide her/him with a structured presentation of the events occurred in the context of each specific collaboration.

- The second perspective is the network of collaborations established within the personal cloud. For instance, in order to support team work and group interaction, the cloud services should share the definition of the collaboration groups and handle the users' workspaces across all their collaborations.

These perspectives can be managed by modeling the Web Desktop as the front-end of an environment which synchronizes the integrated applications with respect to a shared context. Specifically:

- Besides the personal contact lists offered by most collaboration tools, the environment has to manage global collaboration groups, defined and visible at the level of the personal cloud.

- The information awareness stream generated by the cloud services has to be replaced with a global one, administered at the cloud level.

For this purpose, we introduce the concept of activity sphere as the first building block of a personal cloud. The activity sphere represents a collaboration group within the scope of the cloud. Thus, any integrated application must be enabled to reference the defined activity spheres. Moreover, any user participating in collaboration within the cloud must be allowed to access the spheres (s)he belongs to. Notice that a singleton set of users can be associated to a sphere devoted 


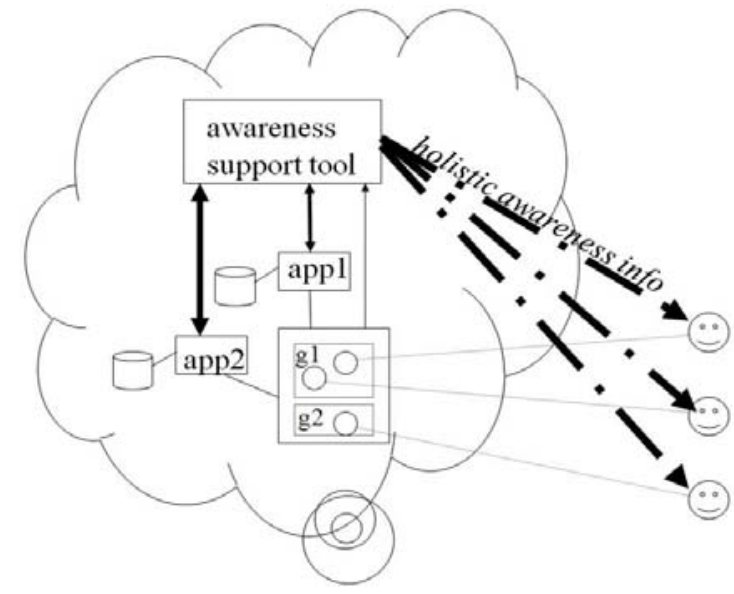

Figure 2. Holistic awareness support in a service cloud.

to the management of the user's private activities by exploiting the cloud services.

In order to offer a user-centric awareness support:

- A personal cloud should synchronize services with respect to the defined activity spheres. Specifically, each application should use, or import, the user groups associated to the spheres. In this way, a user would not need to replicate the definition of her/his collaborations for each business service. Moreover, (s)he could use the spheres as a powerful mean for structuring the visibility scope of her/his workspace across her/his collaborations.

- For each activity sphere, and for each application, the personal cloud should retrieve the awareness information about the activities carried out by the involved users.

- Finally, it should organize such information in order to support its presentation to the users.

The second building block of a personal cloud is therefore a tool supporting the collection of the awareness information generated by the cloud services, and its organization in a structured information flow. Figure 2 depicts this situation: the information streams generated by the applications are redirected to an awareness support tool which absorbs them and organizes the information at the cloud level for presentation purposes.

\section{THE SYNCFR FRAMEWORK}

Before describing the Personal Cloud Platform, we shortly present its underlying infrastructure, i.e., the SYNChronization Framework (SynCFr), which was described in detail in [4].

SynCFr synchronizes heterogeneous services and applications in a shared context. The synchronization is achieved by introducing the Coordination Middleware as an intermediary which supports a loosely-coupled interaction between software components, based on the Shared DataSpace model [19].

Figure 3 shows the framework architecture:

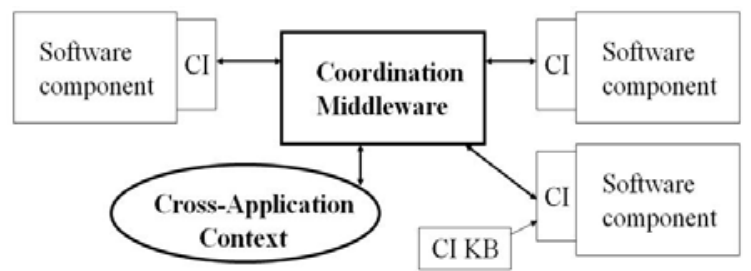

Figure 3. Architecture of the SynCFr framework.

- The Cross-Application Context stores the information to be shared by the components.

- The Coordination Middleware propagates the shared context information among components according to the Publish and Subscribe pattern.

- Each component is wrapped by a Communication Interface (CI), which:

o Subscribes for the context information relevant for the component.

o Supports the execution of the component as an event-driven system [12], on the basis of the events it receives from the Coordination Middleware.

o Captures the events/messages generated by the component and publishes their content by invoking the Coordination Middleware.

SynCFr can be used to integrate heterogeneous services and applications based on Web APIs, REST and WSDL/SOAP interfaces. For instance, it can be exploited to compose Web Services in processes, as in Service Oriented Computing [20]. Moreover, it supports the coordination of event-based Web applications with respect to the user's actions.

The framework is developed in Java and the Coordination Middleware exploits GigaSpaces [9] for the management of the Cross-Application Context and for the subscription-based propagation of events among software components. GigaSpaces is highly scalable and it supports the propagation of data items according to the Linda tuple space model [19]. Being Java-based, it manages the propagation of serializable objects, which can store complex information items.

As described in [4], SynCFr offers a set of skeleton classes for the development of the CIs. However, the administrator of the environment is asked to write the pieces of code needed to establish the connection and to interact with the wrapped components. This code depends on the kind of API offered by the components. For instance, some business applications offer APIs which can be invoked once to receive all the generated events of the specified type; other applications have to be polled by their clients.

\section{The Personal Cloud Platform}

\section{A. Architecture}

The PCP model supports the management of personal clouds offering a basic set of functions to be expanded by integrating the business services needed by the target 
community of users. Figure 4 shows the architecture of the PCP. This is based on the synchronization of a set of components supporting the definition of the activity spheres and the unified management of the awareness information. Specifically:

- The User Agent stores the identities of the registered cloud users and supports the specification of information awareness preferences; e.g., notification preferences. Currently, we are not dealing with privacy preferences, which should be stored by this Agent.

- The Group Manager application supports the definition of the activity spheres and of the associated collaboration groups, which are formed by selecting users registered in the personal cloud.

- The Survey Manager application handles generic types of surveys which the cloud users may have to answer. In particular, it manages the acceptance/rejection answers of users when they are invited to join in an activity sphere.

- The Notification Manager mediates the delivery of notifications to the user. It absorbs the awareness information generated by the cloud applications and it organizes the information flow for presentation purposes; see Section 4.2.3. This component invokes an Instant Messaging Application to send alerts to the users.

- The Instant Messaging (IM) Application supports the synchronous user communication, as well as the management of presence information within the activity spheres.

- The Data Mediator addresses data-interoperability problems within the applications integrated in the platform by applying data-translation rules to generate data items in the format required by the other applications. For instance, we have exploited it to handle data-format issues in the integration of heterogeneous calendar management services. The data Mediator uses the ".ics" format (of the iCalendar standard) and can therefore read calendars that have been exported in this format by other applications, such as Thunderbird.

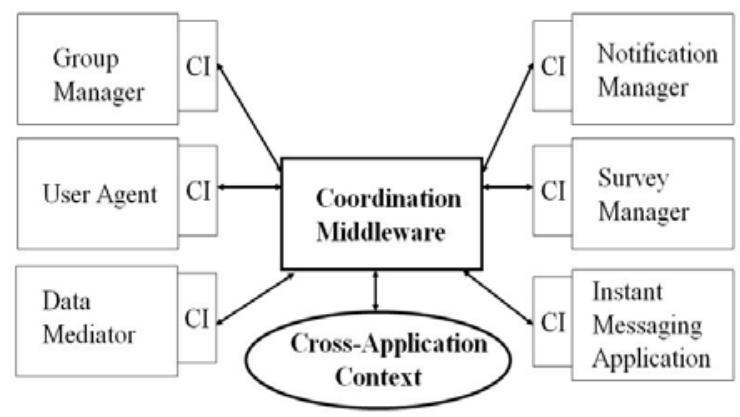

Figure 4. Architecture of the Personal Cloud Platform.

\section{B. Synchronization Model}

The PCP components synchronize by sharing, via their CIs, the context information available in the CrossApplication Context. For this purpose, at personal cloud setup time, each component has to subscribe for the types of events needed to carry out its own activities. At runtime, the components have to publish the events concerning the context information to be shared with the other applications.

The propagation of context information supports both the sharing of general data, such as the definitions of the activity spheres, and the management of complex operations within the personal cloud, possibly involving the execution of processes to which several components participate.

For instance, the data mismatches among applications are addressed as follows: the Data Mediator subscribes for the events concerning the data items to be translated, applies the appropriate translation rules and publishes the translated versions. In turn, the applications interested in such versions have to subscribe for them.

As another example, the creation and the revision of an activity sphere is managed as follows (see Figure 5):

- The Group Manager interacts via User Interface with the user to retrieve the name of the sphere and the list of invited members. Then, it publishes such information in the Cross-Application Context, as a membershipProposal(groupName, userNames) event, where userNames denotes the members to be invited.

- The Survey Manager is subscribed to membershipProposal events. When it receives an event of this type, it starts a survey aimed at asking the involved users to accept or reject the invitation. Meanwhile, it publishes a membershipSurvey(groupName, userNames) event in the Cross-Application Context, as a piece of awareness information to be conveyed to such users.

- The Notification Manager is subscribed to all survey-related events and thus also to the membershipSurvey ones. When it receives the event, it notifies the involved users that they have to answer a survey (by accessing the User Interface of the Survey Manager).

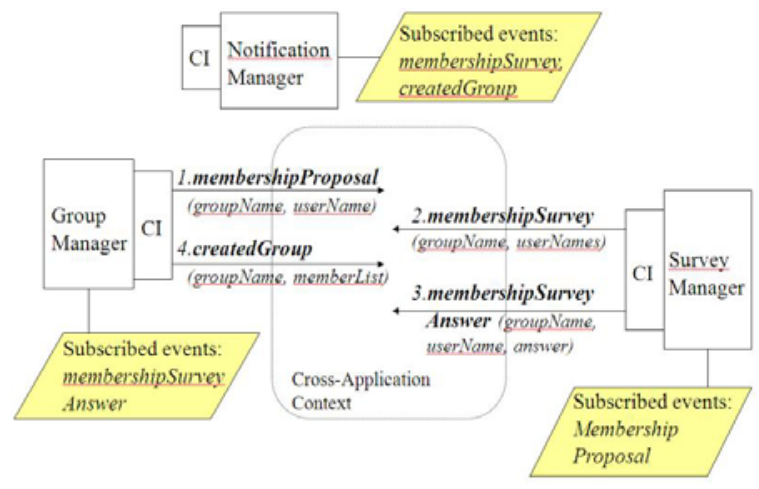

Figure 5. Propagation of context information in the PCP. 
- The Survey Manager collects the user answers and then publishes them in the Cross-Application Context as a set of membershipSurveyAnswer(groupName, UserName, answer) events.

- The Group Manager is subscribed to the membershipSurveyAnswer events. Thus, it retrieves the user's answers from the context and publishes the sphere composition as a createdGroup(groupName, memberList) event.

Within the personal cloud, the applications sharing or importing the activity spheres have to subscribe to the createdGroup events in order to be notified whenever the sphere definitions change.

The personal cloud can be extended with external services by integrating their context information in the Cross-Application Context, provided that such services offer APIs supporting the access to their business data. The services can be either new developed ones, or existing, and possibly heterogeneous ones.

For instance, in order to integrate a document sharing application, the following steps have to be performed:

- The CI of the application has to be developed; see Section 4.1. The CIs for both new developed software components, or for existing external applications, interact with the Coordination Middleware, through a Java library offered by SynCFr.

- At set up time, the CI has to subscribe for the events describing the activity spheres.

- At runtime, the CI has to post the documentmanagement events concerning document uploads, removals, etc., specifying which users share the various documents.

- Moreover, in order to capture the context information generated by the application, the subscription list of the Notification Manager has to be extended with the document-management events.

\section{Web Desktop Support}

The PCP supports the management of Web Desktops offering business services which directly reference the activity spheres defined in the personal cloud. The added value of this approach is twofold: on the one hand, the user is enabled to define her/his collaboration groups only once, and reference them from all the cloud services. On the other hand, the environment mediates the awareness information generated by such services for all the users belonging to the collaboration groups.

Based on the PCP, we developed a prototype personal cloud including a Document Management application, a Calendar Management one, and a Common Calendar application which supports the scheduling of meetings, given the information stored in the calendars of the involved users.

Figure 6 shows a snapshot of the Web Desktop of a user of registered in the personal cloud. The current dashboard is built as an iGoogle page, containing Google Gadgets. This is the best representation of our idea of a Web-based dashboard including views (Gadgets) on the cloud services. However, there is no architectural commitment to use an iGoogle page as the main User Interface of the personal cloud.

The Notification Manager has a central role in the provision of the unified awareness support. In fact, the adoption of a mediated approach to the awareness support enables the introduction of specialized techniques for the delivery and the presentation of information to the user. At the current development stage, the Notification Manager groups the notifications for a user by activity sphere and it presents them in an HTML page supporting the navigation of possibly large amounts of information. For each sphere, the stream of notifications is presented as an HTML page supporting different search criteria (by date, etc., similar to the presentation of e-mail messages). Moreover, the pages concerning different spheres are organized as tabs, so that the user can easily switch among them.

We are currently extending the User Agent and the Notification Manager in order to offer advanced notification policies for the presentation of the awareness information. Specifically, we have defined a context-filter policy which prescribes to deliver the information concerning the user's current activity sphere (tracked by the User Agent), and to delay the other one. If this policy is applied, the Notification Manager sends the non-filtered notifications as Instant Messages; moreover, it presents the delayed notifications as an HTML page organized as described above. Some preliminary tests suggest that the context-filter policy reduces the disruptive effect of notifications on the user. In fact, on the one hand, this policy prevents the user from being overloaded from a continuous flow of unrelated notifications; on the other hand, it guarantees that (s)he is provided with the awareness information relevant for her/his activities, at any time.

\section{TECHNICAL DETAILS}

Our prototype personal cloud is developed in Java, by exploiting the Google Web Toolkit (GWT) to build the User Interface of the applications. The current dashboard is built as an iGoogle page, allowing the users to collect the Gadgets of their favorite apps, as well as those offered by the personal cloud.

The prototype exploits two Google services for the management of the cloud users and of the activity spheres, as these services are natively integrated and thus particularly convenient to use. Specifically:

- All the PCP components employ the Google Authentication service as a single sign-on service. Moreover, they use it to access the business data concerning the registered users; e.g., calendar data.

- The Group Manager application exploits the Google Contacts API to manage the activity spheres as lists of Google accounts. Thus, the members of a group can find in their Contacts the groups they belong to.

However, it should be noticed that the PCP allows the synchronization of heterogeneous applications offering open APIs, therefore there is no architectural bound to use Google APIs or Apps. 
The PCP also includes three applications offering specific collaboration services:

- A Common Calendar application exploits the users' calendars in order to support the scheduling of group meetings.

- Google Docs and Google Calendar are employed as document sharing and calendar management applications.

Specifically, the Common Calendar is a java-based application offering a group meeting scheduling functionality. When a member of a group needs to schedule a meeting, the application reads the calendars of the meeting participants, creates an intersection of the available slots for all of them and proposes a list of possible meeting dates. The user chooses a slot and the application publishes a suitable event specifying the involved group of users. This event is handled analogously to the management of the activity spheres (see Section 4.2.2 and Figure 5), having replaced the Group Manager with the Common Calendar application.

With respect to the integration of other services, the synchronization of the Google applications is simpler because they share some business information, such as the Google Accounts and/or the Contacts lists. However, similar to the general case, such applications have to be wrapped in order to capture the awareness information to be delivered to the users. For instance, we developed the Communication Interface of Google Docs ${ }^{2}$ to capture the events concerning the document uploads and manage them uniformly with the other awareness information generated in the personal cloud.

Indeed, similar to standard Web Desktops, the user is free to add her/his own applications, without integrating them in the personal cloud. However, the awareness support generated by such applications, if any, is handled autonomously. For instance, the Web Desktop shown in Figure 6 includes a GMail Gadget connecting to the GMail service. Although that service shares the user's Google account and the activity spheres stored by the Contacts service, it sends Instant Messages to notify the user about the new e-mail messages, instead of publishing such information in the Cross-Application Context and exploiting the Notification Manager for the presentation of such information to the user.

Before concluding, it is worth mentioning that, although the PCP offers a basic set of components supporting the synchronization of applications within the cloud, the system administrator can use other components for managing the flow of context information within the cloud. For instance, the users are not bound to exploit the Survey Manager for

${ }^{2}$ As Google Docs does not have any callback APIs for event notification, we developed a simple Monitor tracking the user activities on documents. This component keeps a list of documents with some state information and periodically calls the Google API for checking the document status on Google Docs. If the document status has changed or documents have been added or removed, the Document Monitor publishes the appropriate event in the CrossApplication Context. replying to the surveys they are involved in; any application that implements the required Interface for reading and writing events to the Coordination Middleware (e.g., E-mail or SMS clients) could be chosen.

\section{RELATED WORK}

Our work differs from other cloud environments, such as those managed by Amazon and Google, in the following aspects:

- Thanks to the context-based integration of applications, the PCP mediates the presentation of the awareness information to the users and structures it on a contextual basis. Therefore, the user receives a single stream of information, organized on the basis of her/his collaborations, instead of being overloaded by parallel, application-dependent, information flows.

- $\quad$ Being based on an open architecture, the PCP allows the context-based integration of external applications, selected by the personal cloud administrator on the basis of specific community needs.

The context-based integration of applications and the holistic awareness support also differentiates the PCP from the desktop cloud infrastructures, such as [10], which only support the invocation of Web applications as separate resources. Moreover, the goal of our work differs from cloud operating systems, such as [16], which support the development and deployment of cloud applications, as the PCP is aimed at synchronizing existing applications on a contextual basis.

Various e-collaboration tools support file sharing and distributed work among teams or other groups. However, these systems are mostly available as Project Management vertical tools (e.g., [7,1]), or as awareness support tools (e.g., [22]), and cannot be customized to answer the needs of other application domains. In comparison, the PCP enables the users to integrate the awareness support offered by separate e-collaboration tools. Moreover, as already discussed, it can be extended with new applications, therefore supporting the development of customized collaboration environments.

Indeed, there is an on-going effort to integrate the awareness information generated by different collaboration tools; however, this is limited to very specific kinds of applications, such as Web calendars; e.g., see [6].

As the PCP supports the management of Web Desktops, it is worth comparing it with mashup technology. As discussed in [5], mashups offer little support in terms of models and tools for presentation integration. Although some recent work, such as the IBM Sharable code platform [14], attempts to standardize mashup development, it does not support a flexible integration of software components because it does not offer an explicit infrastructure for sharing their context information. Our work addresses this limitation, while maintaining a loosely-coupled integration approach, by managing the Cross-Application Context and the subscription-based propagation of context information. 


\section{CONCLUSIONS}

This paper has presented the Personal Cloud Platform (PCP) for the management of user-centered service clouds, which we define as personal clouds. Different from a standard service cloud, a personal cloud offers a unified perspective on the user's activities, across all the cloud applications, and all her/his collaborations. This is achieved by supporting the definition of global collaboration groups and by holistically managing the awareness support relative to the activity spheres the user is involved in.

Based on the PCP architecture, we developed a prototype personal cloud offering services for group interaction, document sharing, calendar management and meeting scheduling. The current prototype offers the functions for the holistic awareness support but it currently does not deal with trust, security and privacy management, which is part of our future work. In our future work, we will also deal with the User Interface aspect, e.g., in order to improve the look\&feel, and the functions offered by the Gadgets, both for desktop and handheld devices.

\section{REFERENCES}

[1] ActiveCollab. Project management \& collaboration. http://www.activecollab.com/, 2008.

[2] Adobe. Acrobat http://www.adobe.com/products/acrobatconnect/, 2008.

[3] Apple. MobileMe. Push email, push contacts, push calendar. Everything in sync. Anywhere you are. http://www.apple.com/mobileme/, 2009.

[4] L. Ardissono, A. Goy, G. Petrone, and M. Segnan: SynCFr: Synchronization Collaboration Framework. In: Proc. of ICIW 2009, 18-23, Venezia, Italy, 2009.

[5] B. Benatallah, F. Casati, F. Daniel, and M. Matera. A framework for rapid integration of presentation components. In Proc. of WWW'2007, 923-932, Banff, Canada, 2007.

[6] BusyMac. Share calendars the easy way. http://www.busymac.com/, 2009.
[7] Collanos. Products overview: Team enabling professionals. http://www.collanos.com/en/products, 2008.

[8] M. Czerwinski, E. Horvitz, and S. Wilhite, S.: A diary study of task switching and interruptions. In: Proc. of CHI 2004 Conf. on Human Factors in Computing Systems, Vienna, 2004.

[9] GigaSpaces. GigaSpaces SBA. http://www.gigaspaces.com/pr overview.html, 2008.

[10] Gladinet, inc. Gladinet Cloud Desktop. http://www.gladinet.com/, 2009.

[11] A. Grimes, and A. Brush: Life scheduling to support multiple social roles. In: Proc. of CHI 2008, 821-824, Florence, Italy, 2008.

[12] R. Lu and S. Sadiq. A survey of comparative business process modeling approaches. In Proc. of 10th Int. Conf. BIS, LNCS 4439, 82-94, Poznan, Poland, 2007.

[13] G. Mark, and N. Su: Considering Web 2.0 technologies within an ecology of collaborations. In: Proc. of SociUM: Adaptation and Personalisation in Social Systems: Groups, Teams, Communities, 5059, Corfu, Greece, 2007.

[14] E. Maximilien, A. Ranabahu, and K. Gomadam. An online platform for Web APIs and service mashups. IEEE Internet Computing, (September-October):32-43, 2008.

[15] D. McFarlane, and K. Latorella: The scope and importance of human interruption in human-computer interaction design. Human-Computer Interaction 17(1), 1-61, 2002.

[16] Microsoft. Windows http://www.microsoft.com/azure, 2009.

[17] mywebdesktop.net. Your personal and collaborative desktop on the internet. https://www.mywebdesktop.net/, 2009.

[18] OASIS. Web Services Business Process Execution Language. http://www.oasis-open.org/committees /documents. php?wg abbrev=wsbpel, 2005.

[19] G. Papadopoulos and F. Arbab. Coordination models and languages. In Advances in Computers, 329-400. Academic Press, 1998.

[20] M. Papazoglou and D. Georgakopoulos, eds. ServiceOriented Computing, 46. Communications of ACM, 2003.

[21] P. Scupelli, S. Kiesler, S. Fussell, and C. Chen: Project View IM: a tool for juggling multiple projects and teams. In: Proc. CHI'2005, 1773-1776, Portland, Oregon, 2005. 
File Edit View History goookmarks Iools Help

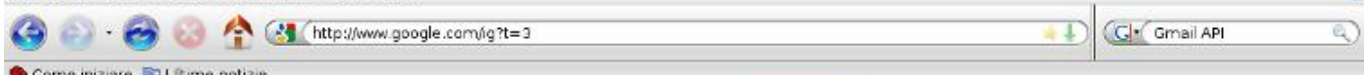

- Come iniziare Elizime notizie

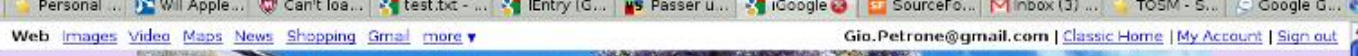

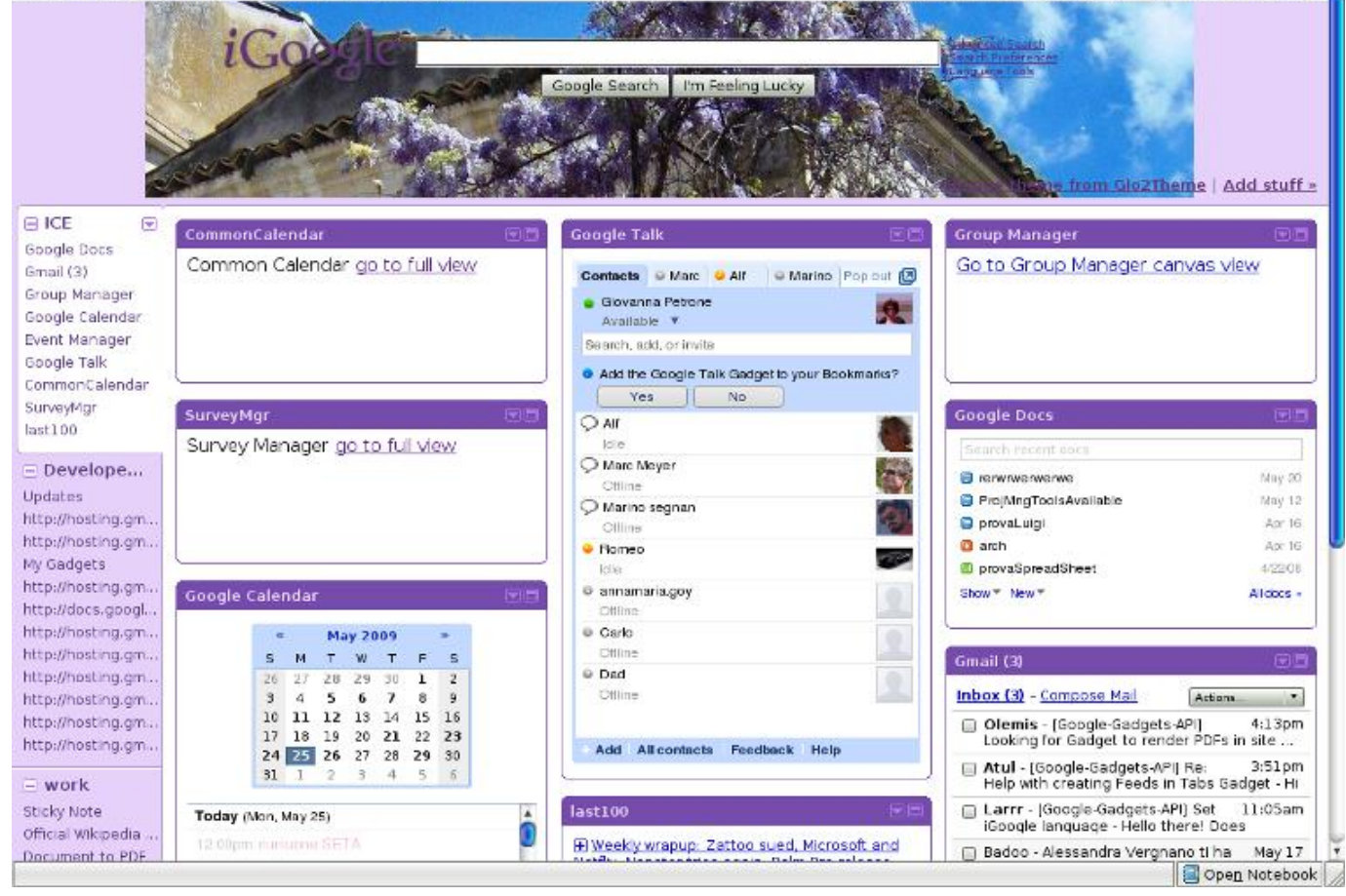

Figure 6. Web Desktop of a user registered in our prototype personal cloud. 